\title{
Ruptured Massive Hepatocellular Carcinoma Cured by Transarterial Chemoembolization
}

\author{
Ji Eun Lee, Joong-Won Park, In Joon Lee, Bo Hyun Kim, Seoung Hoon Kim, Hyun Beom Kim \\ Center for Liver and Pancreatobiliary Cancer, National Cancer Center, Goyang, Korea
}

Received Dec. 27, 2019

Revised Feb. 13, 2020

Accepted Feb. 26, 2020
Spontaneous tumor rupture is a serious but rare complication of hepatocellular carcinoma (HCC) and has a low survival rate. Here, we report a case of massive HCC that ruptured and was treated successfully with transarterial chemoembolization (TACE). A 55-year-old man with abdominal pain was diagnosed with a $12-\mathrm{cm}$-wide ruptured $\mathrm{HCC}$ at segment 8 . The overall liver function was scored as Child-Pugh A, but the single nodule tumor had ruptured; therefore, TACE treatment was initiated. After the first TACE treatment, residual tumors were found; thus, secondary TACE was performed 5 months later. No new lesions or extrahepatic metastases were found 16 months after the first TACE treatment, so hepatic resection was performed for curative treatment. The postoperative pathology results did not reveal any cancer cells; hence, TACE alone resulted in a cure. We report this case because the cure has been maintained for more than 3 years after resection. (J Liver Cancer 2020;20:154-159)

Keywords: Hepatocellular carcinoma; Spontaneous rupture; Transarterial chemoembolization

\section{INTRODUCTION}

Hepatocellular carcinoma (HCC) is the fifth commonest cancer worldwide. ${ }^{1}$ Among all cancers, the mortality rate of HCC is the second highest in Korea. ${ }^{2}$ Probably due to the hypervascularity of HCC, spontaneous tumor rupture is reported in $3-15 \%$ of patients with HCC. ${ }^{3,4}$ This complication is rare but life-threatening, with a 30-day mortality rate of $25-75 \%{ }^{5}$ Fortunately, mortality from the resulting massive bleeding and shock is decreasing due to early diagnosis and effective management. However, long-term survival remains poor because rupture is associated with higher Child-Pugh scores, more advanced staging, and the possibility of perito-

\footnotetext{
Corresponding author : Joong-Won Park

Center for Liver and Pancreatobiliary Cancer, National Cancer Center, 323 Ilsan-ro, Ilsandong-gu, Goyang 10408, Korea

Tel. +82-31-920-1605, Fax. +82-31-920-1520

E-mail; jwpark@ncc.re.kr

https://orcid.org/0000-0001-9972-0494
}

neal seeding from the ruptured tumor. ${ }^{3,6}$ Herein, we present a case of massive ruptured HCC with complete response to transarterial chemoembolization (TACE) without recurrence or metastasis. The need for institutional review board approval was waived for the present study.

\section{CASE REPORT}

\section{Clinical findings}

A 55-year-old man presented at our hospital emergency room with a single large hepatic mass found on an abdominal computed tomography (CT) scan obtained 10 days prior at another hospital for abdominal pain. At our hospital, he did not present with any symptoms, such as abdominal pain. His vital signs were stable and there were no specific abnormal findings on physical examination. He had been diagnosed with hepatitis B 20 years earlier but had taken no anti- 
viral medication. He had consumed a bottle of alcohol once a week and half a pack of cigarettes for 20 years. Initial laboratory tests revealed a hemoglobin level of $13.4 \mathrm{~g} / \mathrm{dL}$, an albumin level of $4.5 \mathrm{~g} / \mathrm{dL}$, a total bilirubin level of $1.1 \mathrm{mg} / \mathrm{dL}$, a prothrombin time international normalized ratio of 0.93 , an aspartate aminotransferase (AST) level of 35 IU/L, and an alanine aminotransferase (ALT) level of 55 IU/L. His serum alpha-fetoprotein (AFP) level was $4.3 \mathrm{ng} / \mathrm{mL}$ and protein-induced vitamin $\mathrm{K}$ absence-II (PIVKA-II) level was 11,485

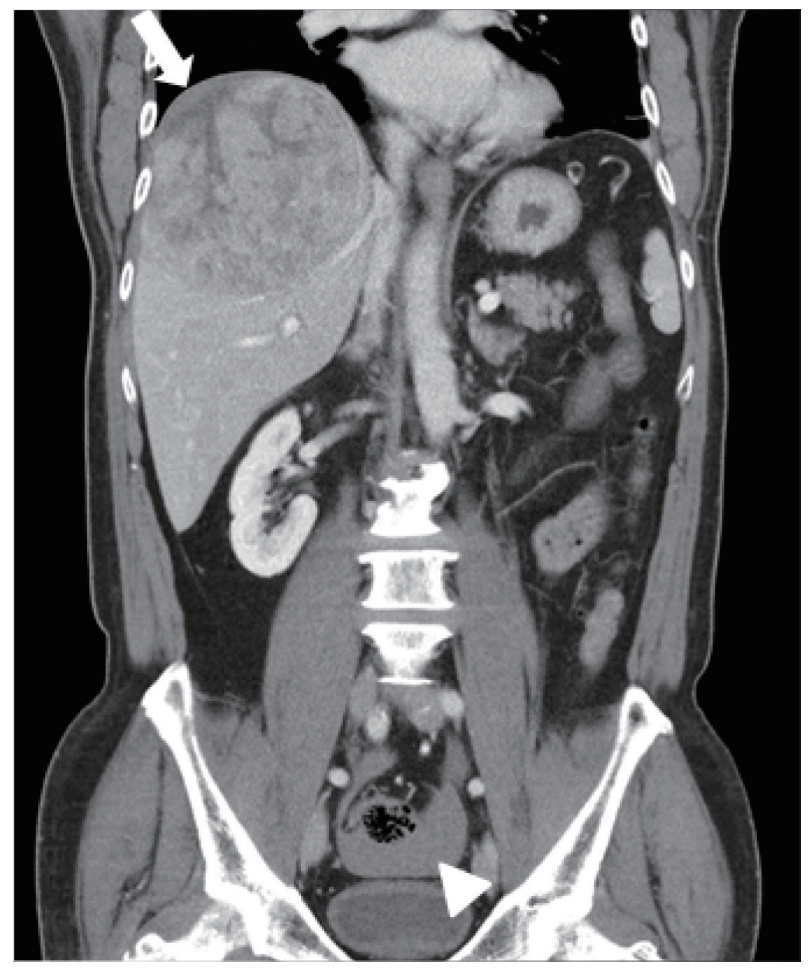

Figure 1. Initial abdominal computed tomography findings. A large hepatic mass was identified at segment 8 . The focal hypo-attenuated lesion on the surface of the mass (arrow) and the fluid collection in the pelvic cavity (arrowhead) indicated a relatively recent tumor rupture.
$\mathrm{mAU} / \mathrm{mL}$. Tests for hepatitis B surface antigen and hepatitis B surface antibody yielded positive and negative results, respectively. His hepatitis B virus DNA level was $36 \mathrm{IU} / \mathrm{mL}$. The anti-hepatitis $C$ virus test result was negative.

\section{Imaging findings}

The abdominal CT scan (performed at another hospital) revealed a large single hepatic mass at segment 8 . A suspected focal and hypoattenuated rupture site was noted on the surface of the mass. In addition, fluid (density of 40-50 Hounsfield units) had collected in the pelvic cavity, suggestive of a hemoperitoneum and indicating a relatively recent tumor rupture (Fig. 1). No extrahepatic metastases were found. Magnetic resonance imaging was also performed and confirmed the presence of a single 12-cm-wide HCC at segment 8 without vascular invasion (Fig. 2). There was no evidence of ongoing bleeding from the rupture site in these images.

\section{Diagnosis and treatment}

The patient's overall performance was good with an Eastern Cooperative Oncology Group score of 0, and his liver function was preserved with a Child-Pugh classification of A. The ruptured tumor was a single nodule without vascular invasion or extrahepatic spread. It was categorized as stage II using the modified International Union Against Cancer system, and intermediate stage using the Barcelona Clinic Liver Cancer (BCLC) system. Based on these results, we started treatment with TACE.

TACE was performed via super-selected feeder vessels us-
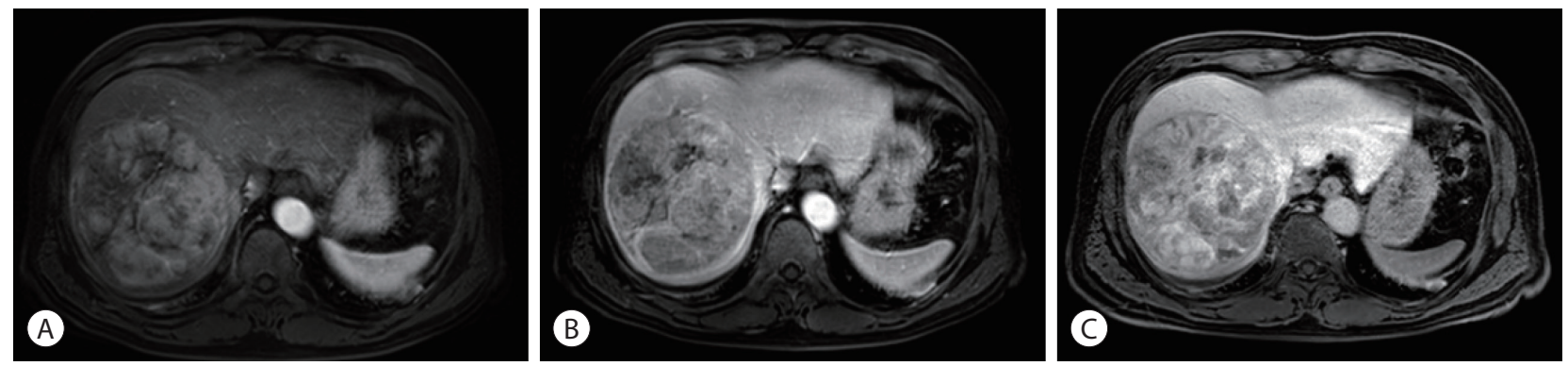

Figure 2. Magnetic resonance imaging findings. A single $12 \mathrm{~cm}$ wide hepatocellular carcinoma lesion at segment 8 without vascular invasion was identified with enhancement in the arterial phase (A), and washout in the portal phase (B) and delayed phase (C). 
ing a 10-mL lipiodol and 50-mg doxorubicin mixture with small polyvinyl alcohol particles to enhance the ischemic effects. After the procedure, the patient developed fever and elevated AST/ALT level for 3 days as part of postembolization syndrome, which resolved with conservative management. Follow-up imaging was performed at one month after TACE, and there was no definite evidence of remnant or recurrent tumor with an AFP level of $4.0 \mathrm{ng} / \mathrm{mL}$ and a PIVKA-II level of $26 \mathrm{mAU} / \mathrm{mL}$. However, the residual tumor was detected at the margin of the treated mass 5 months later and assessed for partial response according to the modified Response Evaluation Criteria in Solid Tumors criteria. Secondary TACE was performed for that lesion (Fig. 3), after which complete response was maintained. The level of AFP and PIVKA-II after secondary TACE were $3.4 \mathrm{ng} / \mathrm{mL}$ and $28 \mathrm{mAU} / \mathrm{mL}$, respectively. We planned additional surgical resection after secondary TACE because surgery is the cura- tive treatment for single nodule without any lymphovascular invasion and distant metastasis. In addition, the liver function of our patient was classified as Child-Pugh A and BCLC stage $B$, which was good enough to administer additional treatment. No new lesions or extrahepatic metastases were found for 16 months after the first TACE. Thus, the patient underwent radical right hemihepatectomy for curative treatment. A single yellow mass in the resected specimen proved to be an area of complete necrosis, with no cancer cells observed in the postoperative pathology examination (Fig. 4), indicating that cure had been achieved by TACE alone. One month after surgery, the serum level of AFP was $3.6 \mathrm{ng} / \mathrm{mL}$, which did not represent much change; however, the level of PIVKA-II decreased to $10 \mathrm{mAU} / \mathrm{mL}$. The hepatitis B virus DNA level had remained inactive at low levels until October 2017, and then increased to $297 \mathrm{IU} / \mathrm{mL}$. The patient started taking entecavir after which the DNA level decreased to less
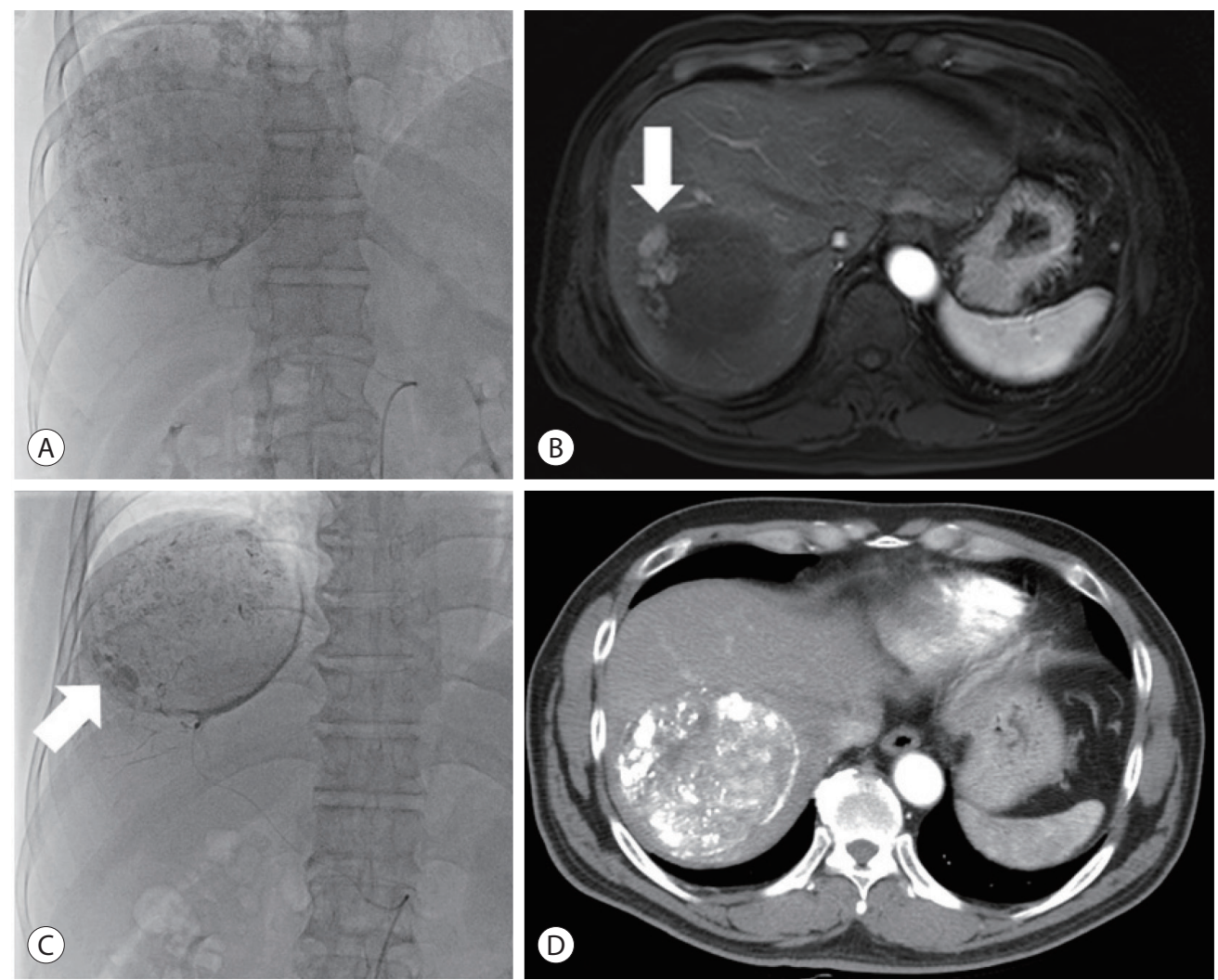

Figure 3. Angiogram of the first transarterial chemoembolization (TACE) showed a single massive tumor staining (A). After the first TACE, residual tumor (arrow) was found on the magnetic resonance imaging (B), and secondary TACE was performed 5 months later. Peripheral tumor staining (arrow) was identified at angiography of the secondary TACE (C). After secondary TACE, the treated mass without residual tumor was noted on the liver computed tomography scan (D). 
than $15 \mathrm{IU} / \mathrm{mL}$. The patient was followed up every 3-6 months without any tumor recurrence or metastasis for more than 3 years after resection.

\section{DISCUSSION}

Spontaneous rupture of HCC is an uncommon and sometimes fatal complication with an incidence rate of 3-15\%. As recent screening of patients at a high risk for HCC has improved, the incidence has decreased steadily. ${ }^{7}$ However, mortality due to spontaneous rupture is the third commonest cause of HCC-related death after tumor progression and liver failure, ${ }^{8}$ and the 30 -day mortality rate is $25-75 \%{ }^{5,9}$ The poor survival rate of patients with ruptured HCC is related to a higher Child-Pugh score, more advanced staging, and possible peritoneal seeding from the ruptured tumor. ${ }^{3,5,6,10}$ The remarkable prognosis in this case might have been due to the patient's good liver function (Child-Pugh class A) and the relatively low HCC stage (BCLC B) at the time of treatment. A recent nationwide study performed in Japan reported that the 1-, 3-, and 5-year overall survival rates among patients with ruptured HCC were $41.4 \%, 21.1 \%$, and $13.3 \%$, respec- tively, which were worse than those among HCC patients without rupture. ${ }^{11}$

Previous studies have shown that the risk factors for HCC rupture are tumor location on the hepatic surface, tumor diameter $>5 \mathrm{~cm}$, portal hypertension, and liver cirrhosis. ${ }^{3,8,12}$ One of the most relevant factors is peripheral surface location and/or protrusion of the tumor. ${ }^{3}$ In our case, the $12 \mathrm{~cm}$ HCC tumor was located at the periphery of segment 8 , and the suspected rupture site was on the surface of the tumor just below the diaphragm, consistent with the risk factors identified in previous studies. Although the exact mechanisms underlying spontaneous HCC rupture are not known, friable feeding vessels and a tear at the surface of the tumor, which are associated with a small amount of pressure caused by diaphragmatic movement during respiration or increased intratumoral pressure caused by rapid tumor growth are all thought to be possibilities. ${ }^{5,13}$

The most common symptom of HCC rupture is acute abdominal pain, which occurs in $66.4 \%$ of patients. ${ }^{14}$ In this case, the patient's abdominal pain occurred suddenly 10 days prior, prompting a visit to another hospital, and then resolved by the time he presented to our hospital. We conclud-
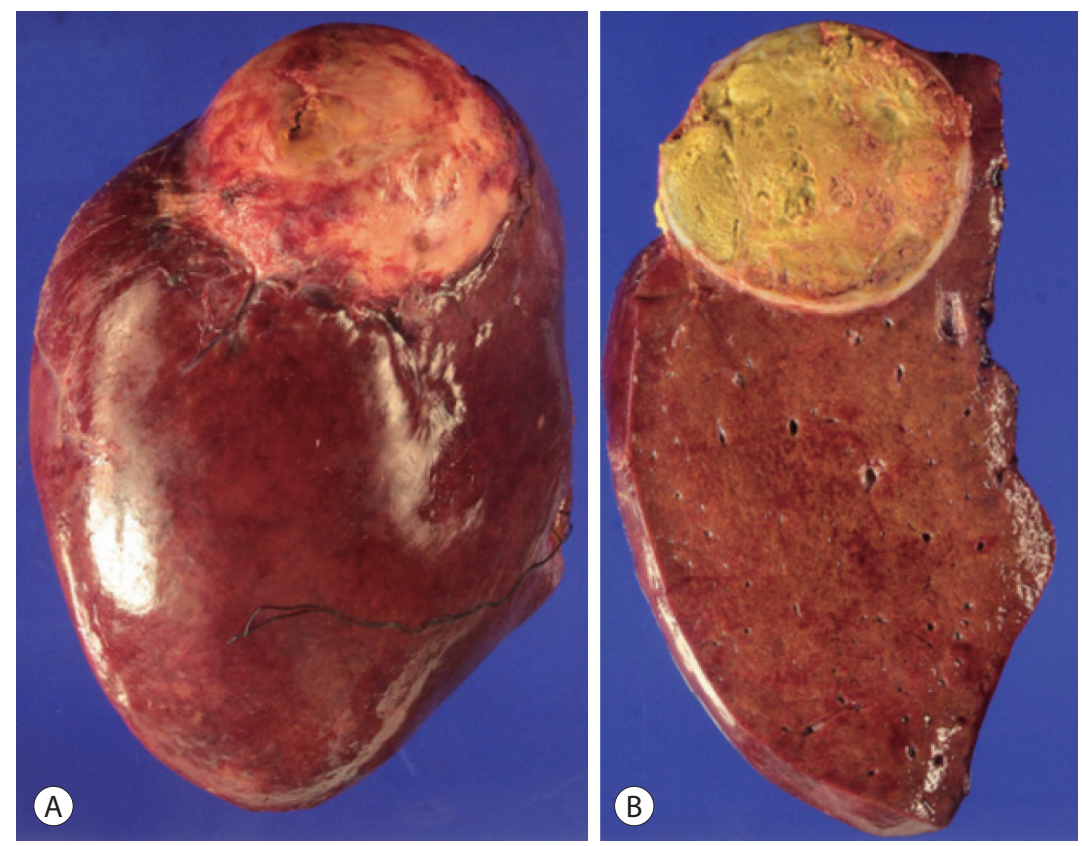

Figure 4. The exterior ( $A$ ) and interior (B) of the segment $7 / 8$ tumor resected by right hemihepatectomy. The single yellow mass proved to be complete necrosis with no cancer cells. 
ed that the HCC had ruptured 10 days prior, followed by spontaneous hemostasis. Shock and abdominal distension have also been reported in $6.7 \%$ and $16 \%$ of cases, ${ }^{14}$ respectively, but were not present in this case.

The median survival duration after HCC rupture is only 1.2-4 months when not treated. ${ }^{15}$ With treatment, survival has been reported to vary depending on treatment type, with TACE and liver resection being associated with better posttreatment survival than conservative supportive care. ${ }^{9,13}$ TACE is the preferred treatment modality, compared with transarterial embolization, due to its additional benefit of chemotherapeutic and embolic effect to HCC. Moreover, TACE is considered a suitable treatment to achieve hemostasis, and even a definitive cure, if super-selective TACE is feasible. ${ }^{8}$ Liver resection may be an option in patients with preserved liver function and resectable tumors. Although several studies have shown that survival rates are not significantly different between TACE and surgical resection, ${ }^{9,16}$ Aoki et al. ${ }^{11}$ reported that survival was better after surgical resection than after other treatment modalities. Many investigators have reported that it is reasonable to perform TACE as a primary treatment for ruptured HCC, followed by staged hepatectomy once the patient is stabilized. ${ }^{5,16,17}$ In our patient, TACE via super-selected feeder vessels was performed, and the final pathological finding from examination of the surgical specimen was only necrotic tissue without any cancer cells, suggesting that a definitive cure had been achieved via TACE.

Contrary to our expectations, HCC rupture does not appear to increase the risk of metastasis. According to Lee et al. ${ }^{17}$, peritoneal recurrence rates were not significantly different between ruptured HCC and non-ruptured HCC at $14.3 \%$ and $10.0 \%$, respectively. This may be explained by the lack of nourishing vessels necessary for HCC growth in the peritoneum ${ }^{7}$ and/or the patient's death before the metastasis becomes evident clinically. ${ }^{18}$ However, several cases of peritoneal metastasis after HCC rupture have been reported, and the prognosis was extremely poor in some cases, ${ }^{6,7,7,19}$ with a time to metastasis of 8 months. ${ }^{20}$ In our case, no new lesions or extrahepatic metastases were found for 16 months after the first TACE, and right hemihepatectomy was performed for curative treatment. This complete response had been maintained for more than 3 years after surgical resection.

In conclusion, we presented a case of massive HCC rupture, successfully cured for more than 3 years after TACE and surgical resection. We therefore suggest that TACE may be a useful treatment option after HCC rupture.

\section{Conflicts of Interest}

The authors have no conflicts to disclose.

\section{REFERENCES}

1. Bray F, Ferlay J, Soerjomataram I, Siegel RL, Torre LA, Jemal A. Global cancer statistics 2018: GLOBOCAN estimates of incidence and mortality worldwide for 36 cancers in 185 countries. CA Cancer J Clin 2018;68:394-424.

2. Korean liver Cancer association, National Cancer Center. 2018 Korean Liver Cancer Association-National Cancer Center Korea practice guideline for the management of hepatocellular carcinoma. Gut Liver 2019;13:227-299.

3. Zhu Q, Li J, Yan JJ, Huang L, Wu MC, Yan YQ. Predictors and clinical outcomes for spontaneous rupture of hepatocellular carcinoma. World J Gastroenterol 2012;18:7302-7307.

4. Kim YI, Ki HS, Kim MH, Cho DK, Cho SB, Joo YE, et al. Analysis of the clinical characteristics and prognostic factors of ruptured hepatocellular carcinoma. Korean J Hepatol 2009;15:148-158.

5. Lai EC, Lau WY. Spontaneous rupture of hepatocellular carcinoma: a systemic review. Arch Surg 2006;141:191-198.

6. Chan WH, Hung CF, Pan KT, Lui KW, Huang YT, Lin SY, et al. Impact of spontaneous tumor rupture on prognosis of patients with T4 hepatocellular carcinoma. J Surg Oncol 2016;113:789-795.

7. Uchiyama H, Minagawa R, Itoh S, Kajiyama K, Harimoto N, Ikegami $T$, et al. Favorable outcomes of hepatectomy for ruptured hepatocellular carcinoma: retrospective analysis of primary R0hepatectomized patients. Anticancer Res 2016;36:379-385.

8. Bassi N, Caratozzolo E, Bonariol L, Ruffolo C, Bridda A, Padoan L, et al. Management of ruptured hepatocellular carcinoma: implications for therapy. World J Gastroenterol 2010;616:1221-1225.

9. Jin YJ, Lee JW, Park SW, Lee JI, Lee DH, Kim YS, et al. Survival outcome of patients with spontaneously ruptured hepatocellular carcinoma treated surgically or by transarterial embolization. World J Gastroenterol 2013;19:4537-4544.

10. Leung CS, Tang CN, Fung KH, Li MKW. A retrospective review of transcatheter hepatic arterial embolization for ruptured hepatocellular carcinoma. J R Coll Surg Edinb 2002;47:685-688.

11. Aoki T, Kokudo N, Matsuyama Y, Izumi N, Ichida T, Kudo M, et al. Prognostic impact of spontaneous tumor rupture in patients with hepatocellular carcinoma: an analysis of 1160 cases from a nation- 
wide survey. Ann Surg 2014;259:532-542.

12. Li J, Huang L, Liu CF, Cao J, Yan JJ, Xu F, et al. Risk factors and surgical outcomes for spontaneous rupture of BCLC stages $A$ and B hepatocellular carcinoma: a case-control study. World J Gastroenterol 2014;20:9121-9127.

13. Sahu SK, Chawla YK, Dhiman RK, Singh V, Duseja A, Taneja S, et al. Rupture of hepatocellular carcinoma: a review of literature. J Clin Exp Hepatol 2019;9:245-256.

14. Miyamoto M, Sudo T, Kuyama T. Spontaneous rupture of hepatocellular carcinoma: a review of 172 Japanese cases. Am J Gastroenterol 1991;86:67-71.

15. Al-Mashat FM, Sibiany AM, Kashgari RH, Maimani AA, Al-Radi $A O$, Balawy IA, et al. Spontaneous rupture of hepatocellular carcinoma. Saudi Med J 2002;23:866-870.

16. Lee HW, Lim CS, Kim HS. Outcomes of surgical resection for rup- tured hepatocellular carcinoma. J Liver Cancer 2017;17:54-59.

17. Lee HS, Choi GH, Kang DR, Han KH, Ahn SH, Kim DY, et al. Impact of spontaneous hepatocellular carcinoma rupture on recurrence pattern and long-term surgical outcomes after partial hepatectomy. World J Surg 2014;38:2070-2078.

18. Yunoki Y, Takeuchi H, Makino Y, Murakami I, Yasui Y, Tanakaya K, et al. Intraperitoneal seeding of ruptured hepatocellular carcinoma: case report. Abdom Imaging 1999;24:398-400.

19. Lin CC, Chen CH, Tsang YM, Jan IS, Cheu JC. Diffuse intraperitoneal metastasis after spontaneous rupture of hepatocellular carcinoma. J Formos Med Assoc 2006;105:577-582.

20. Kaido T, Arii S, Shiota M, Imamura M. Repeated resection for extrahepatic recurrences after hepatectomy for ruptured hepatocelIular carcinoma. J Hepatobiliary Pancreat Surg 2004;11:149-152. 